\title{
EAl Endorsed Transactions

\section{Paradoxes in Eye Health Care in Low-Income Countries and Design Strategies for Mobile Health Technology to Overcome them}

\author{
Esmael Kedir Nida ${ }^{1, *}$, Luc Geurts $^{1}$ and Vero Vanden Abeele ${ }^{1}$ \\ ${ }^{1}$ KU Leuven, e-Media lab, Vesaliusstraat 13, 3000 Leuven, Belgium
}

\section{Abstract}

INTRODUCTION: Low income countries bear $90 \%$ of the burden of avoidable blindness. In particular, glaucoma is one of the major causes of visual impairment. Early detection is critical to avoid irreversible blindness due to this underdiagnosed disease. Mobile and connected technologies yield potential to support early detection. However, to realize this potential, they should be designed from a deep understanding of the needs of the local population, and the challenges unique to lowincome countries.

OBJECTIVES: To gain a contextualized understanding of eye health care needs, and the challenges and opportunities for designing mobile health solutions for screening of glaucoma.

METHODS: We conducted a qualitative user study at three different healthcare centres in Southwest Ethiopia, including semi-structured interviews with eight eye care health professionals and observations and informal interviews with 125 patients, at three different locations. Interview transcripts were analysed through inductive thematic analysis involving three iterations.

RESULTS: Seven themes were initially identified after two iterations. The themes provided insight into the organization of eye health care and highlighted the challenges faced by eye care professionals and patients. An additional of three themes were identified in the third iteration which were formulated as paradoxes("Money is not an issue, yet there is no money for glaucoma", "Shortage of eye health care, yet the work is repeated over and over", "The willing but uncooperative patient tested by the willing but unexperienced nurse").

CONCLUSION: Findings from this research bear the potential to inspire future designs of mobile health solutions targeting rural communities in Ethiopia and other LICs.

Keywords: ICT4D, mobile health technology, eye health care, glaucoma, qualitative user research.

Received on 05 December 2018, accepted on 29 January 2019, published on 12 February 2019

Copyright (0) 2019 Esmael Kedir Nida et al., licensed to EAI. This is an open access article distributed under the terms of the Creative Commons Attribution licence (http://creativecommons.org/licenses/by/3.0/), which permits unlimited use, distribution and reproduction in any medium so long as the original work is properly cited.

doi: 10.4108/eai.30-10-2018.161366

\section{Introduction}

According to the World Health Organization, 285 million people worldwide suffer from visual impairment, of which 39 million are fully blind [1]. About $80 \%$ of the visual impairments are due to curable or preventable causes [2]. Low-income countries (LICs) bear a large proportion of the burden of avoidable blindness [1]. Ethiopia is such a LIC with a high prevalence of visual impairment and blindness
[3]. Glaucoma is one of the major causes of irreversible blindness in Ethiopia [4]. According to a national survey in 2006, the disease caused blindness to an estimated 62,000 people [4]. Worldwide, glaucoma is the third major cause of blindness, following cataract and trachoma. With respect to causes of irreversible blindness, glaucoma is the leading cause [5]. Early detection of glaucoma is critical to avoid irreversible blindness [6]. If detected early, further

\footnotetext{
*Corresponding author. Email:esmaelkedir.nida@kuleuven.be
} 
progression of glaucoma can be arrested through antiglaucoma medication or surgery [5].

Mobile technologies are increasingly being utilized in health care in low-income settings, characterized by a limited access to standard diagnostic equipment [7-9]. Hence, they provide the potential for the promotion of early glaucoma detection. However, for mobile health technology to be successful, it needs to address the needs of the local population, and deal with challenges which are unique to LICs, such as inadequate infrastructure, low literacy level of health professionals and unsupportive cultural practices $[10,11]$. We argue for the design of eye health care technologies based on the situated needs of LICs and the systematic inclusion of stakeholders[12].

We present results from a qualitative user study including on-site visits, observations, informal and semi-structured interviews at three different health centre locations in Southwest Ethiopia. Our results provide insight into the organization of eye care in Ethiopia and the needs of the local stakeholders. While the study is situated in Southwest Ethiopia and has a special emphasis on mobile health solutions to aid with the screening of glaucoma we, expose causal mechanisms behind current paradoxes that may typify eye health care in other low-income regions. Furthermore, this work may also contribute to the design of future pervasive healthcare solutions, by highlighting design opportunities to overcome these paradoxes.

\section{Related Work}

\subsection{Glaucoma and Eye Healthcare in Ethiopia}

Glaucoma is an umbrella term for a group of diseases that lead to progressive damage to the optic nerve. It is commonly caused by fluid that builds up in the front part of the eye, increasing the internal pressure and subsequently damaging the optic nerve [13]. Vision loss due to glaucoma initially occurs at the peripheral field of vision and gradually advances to the central vision. Patients are not aware of the condition until there is a significant vision loss, which is why it is also referred to as "the silent thief of sight" [14].

Unfortunately, people in rural areas of Ethiopia need to travel long distances to larger urban centres to gain access to eye care [15]. Similar to healthcare systems in several other countries, the Ethiopian system is organized into three tiers (see Figure 1). The primary level aims to deliver local care. The secondary level entails the care provided by medical specialists. Finally, the tertiary level involves specialized hospital care [16].
However, the population per Ethiopian health care facility is three times higher than even the sub-Saharan average; indicating a tremendously more challenged infrastructure compared to high income countries [17]. There is also a significant shortage of health professionals in the health system. In the year 2002, the ophthalmologists to population ratio in Ethiopia was one ophthalmologist per million people, with more than $70 \%$ of them working in Addis Ababa, the capital [15]. This low prevalence of eye health care professionals stands in sharp contrast with the prevalence of eye diseases, which are even more concentrated in rural areas [3].

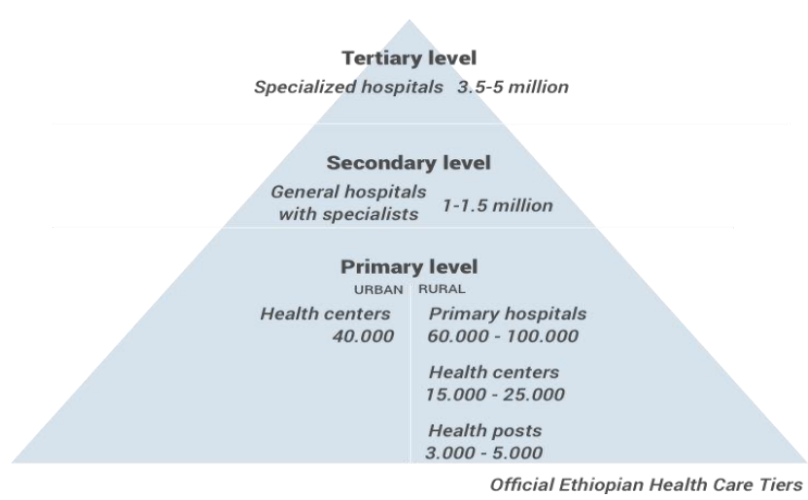

Figure 1. Health Tier System of Ethiopia (Health Sector Development Program IV] [18])

Given the asymptomatic nature of glaucoma and the lack of access, most patients from rural areas visit eye care centres only once they experience a palpable loss of vision[19]. Such patients with advanced glaucoma face difficulty in various activities like reading, noticing objects in their peripheral vision and adapting to different levels of lighting [20]. Besides vision loss, the resulting dependence on others, diagnostic concerns as well as the costs and potential side effects associated with treatments often negatively impact quality of life [21].

\subsection{Screening tests and technologies}

To screen for glaucoma, three tests are performed [6]. First, intra-ocular pressure is measured using a tonometer. Second, the optic disc is inspected using an ophthalmoscope. Finally, the visual field is inspected to check for areas of vision loss, mostly using standard automated perimetry (SAP) equipment [22]. Referral decisions for further diagnosis of glaucoma are based on the combined results of these three tests [6].

Handheld and portable versions of both tonometer and ophthalmoscope are widespread in Ethiopia, and used for community screening in situations where standard hospital-based instrument are not available [23]. These instruments operate without electricity, making them well 
suited for screening, even in remote rural areas where there is limited or no access to electricity. However, for visual field testing (VFT),such an electricity independent, portable instrument is not yet available.

\subsection{Mobile health technologies and screenings}

The availability of wireless communication with mobile devices in LICs has leapfrogged conventional landline systems [24]. The use cases of feature phones and smartphone applications in health care include patient assessment and education, physician reference and education, photographic and image processing and patient record keeping. For instance, there are a number of mobile applications for visual acuity testing that can be used in situations where other tests of visual acuity are not readily available [25]. Smartphones are also being utilized for retina imaging with an indirect lens and the built-in camera [26]. Assessment of refraction with a pinhole adopter attached to a smartphone is also another example to mention [27].

\section{Method}

We conducted a qualitative user study at three different healthcare centres in Southwest Ethiopia, with the main aim of gaining a contextualized understanding of eye health care needs, and the challenges and opportunities for designing mobile health solutions for screening of glaucoma. To this end, we carried out semi-structured interviews with health professionals, as well as informal interviews and observations with patients. Questions in the semi-structured interviews centred on gaining knowledge about the current practices of glaucoma screening and diagnosis. Following patient registration, screening, and diagnosis allowed us to observe different contexts during consultation hours as well as during an outreach mission, an organized field trip of eye healthcare workers to rural areas.

\subsection{Duration and Location}

During July and August 2017, we collected data in three locations in Southwest Ethiopia (see Figure 2). Jimma University Medical Center (JUMC) is a hospital located in the city of Jimma, $350 \mathrm{~km}$ southwest of the capital Addis Ababa, in the Oromia regional state. Seka Chokorsa Primary Hospital (SCPH) is part of the small town of Seka, $23 \mathrm{~km}$ from Jimma, also in the Oromia regional state. Finally, Saja Primary Hospital (SaPH) sits in in the town of Saja, about $110 \mathrm{~km}$ on the main road from Jimma to Addis Ababa, in the 'Southern Nations, Nationalities and Peoples' regional state.

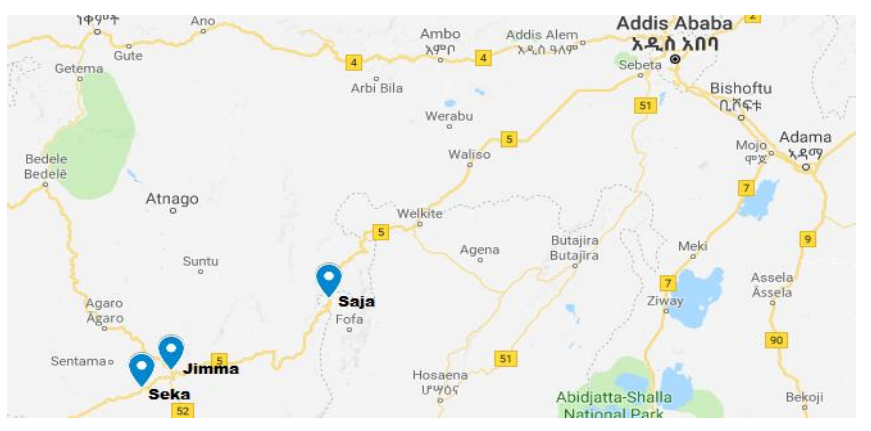

Figure 2. Locations of hospitals included in the study. Saja is $240 \mathrm{~km}$ Southwest of Addis Ababa, Jimma is $350 \mathrm{~km}$ Southwest of Addis Ababa and Seka is $373 \mathrm{~km}$ Southwest of Addis Ababa.

\subsection{Participants}

The eight participants we observed (see Table 1) included two ophthalmologists, three ophthalmic nurses and three Integrated Eye Care Worker (IECW) nurses. IECW nurses are general nurses who received a short training on the treatment of Trachoma Trachealis (TT), a disease caused by an infection resulting in inflammation and scarring of the surface of the eye [28]. We recruited participants through snowball sampling, a technique where each participant suggests further potential interview partners [29]. All semi-structured interviews happened on site and in a one-on-one setting, except one interview which was conducted with two IECW nurses at the same time. In addition to the semi-structured interviews, we informally conversed with patients. At JUMC, we observed the diagnosis of about ten glaucoma patients. During the outreach mission at $\mathrm{SaPH}$ we encountered 115 patients, screened over two days.

Table 1. Overview of participants in the semistructured interviews.

\begin{tabular}{|l|l|l|}
\hline Center & & Occupation \\
\hline \multirow{4}{*}{$\begin{array}{l}\text { Jimma University } \\
\text { Medical Center (JUMC) }\end{array}$} & P1 & Ophthalmologist \\
\cline { 2 - 3 } & P2 & Ophthalmologist \\
\cline { 2 - 3 } & P3 & $\begin{array}{l}\text { Outreach } \\
\text { Coordinator }\end{array}$ \\
\cline { 2 - 3 } & P4 & Ophthalmic Nurse \\
\hline $\begin{array}{l}\text { Saja Primary Hospital } \\
\text { (SaPH) }\end{array}$ & P5 & Ophthalmic Nurse \\
\cline { 2 - 3 } & P6 & IECW Nurse \\
\hline $\begin{array}{l}\text { Seka Chokorsa Primary } \\
\text { Hospital (SCPH) }\end{array}$ & P7 & IECW Nurse \\
\cline { 2 - 3 } & P8 & IECW Nurse \\
\hline
\end{tabular}

\subsection{Data Analysis}

All interviews and observations were conducted by the first author of the paper.All interviews were audio-recorded and transcribed immediately. Next, a thematic analysis was carried out involving two additional researchers. according 
to an inductive thematic analysis process [30]. No a priori lenses or theories were established, but rather themes were identified via the data. In a first iteration, in vivo coding was applied by each researcher individually to generate initial codes after familiarizing with the data. In a second iteration, initial codes were revisited and searched for themes describing trends. Codes were clustered into concepts, and core concepts were defined for each cluster via the affinity diagramming technique [31]. In a final iteration, themes were revisited and three additional themes, which were formulated as paradoxes, were added.

\section{Results}

We have identified seven themes in our thematic analysis. These provide insights in the organization of eye health care and the needs of local stakeholders.

Tertiary health care to provide primary eye health care Our first theme concerns the provision of health care according to the different layers of primary, secondary and tertiary care. We found that eye care beyond treating infections and TT is not well integrated into the primary level of health care. Hospitals and health centers lack the necessary equipment and ophthalmic professionals to provide basic eye care services. As a consequence, patients who need eye care are required to travel to a more distant and less accessible specialized hospital. Not all patients can afford to travel such distances. Therefore, sometimes, primary hospitals prescribe medication for glaucoma without a proper diagnosis.

Observations. SCPH, as a primary hospital, is expected to serve up to 100.000 patients. Despite the high prevalence of eye diseases, we found there neither a dedicated room, nor a specifically educated eye care professional besides two IECW nurses. These provide treatment for patients with TT on site, in addition to their regular duties. A patient with any other eye problem is referred to JUMC, even when it is known that patients will not be able to do so. The nurses consider glaucoma to be beyond their expertise as they lack the necessary training and equipment.

P7: We take the history and visual acuity. If it is an infection, we give them antibiotics. If we see TT, we do surgery for them. But if it is cataract, we refer them to Jimma. But some of them don't go to

Jimma because they cannot afford it

$\mathrm{SaPH}$ serves a similar number of patients as SCPH. Here, among the two nurses one is specialised on ophthalmology. A small room serves as an eye unit; however, it is poorly equipped. Besides a Snellen chart to test visual acuity, the room contains one handheld tonometer to measure the intraocular pressure (IOP) and a torch light, used as an ophthalmoscope(see Figure 3).
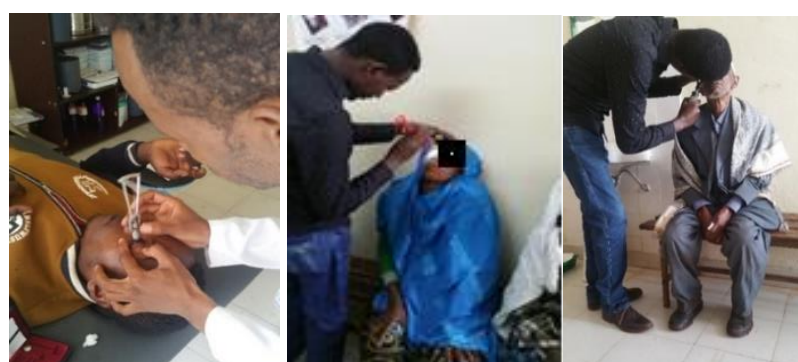

Figure 3: An ophthalmic nurse measuring intraocular pressure (left) and screening the optic disc, using torch light (middle) and a handheld ophthalmoscope (right).

The medical staff has no other instruments to further eye examinations available. Similar to SCPH, beyond eye allergies and infections curable with medication, TT is the only eye condition that can be treated at this hospital. For most other eye diseases including glaucoma, patients are referred to JUMC.

\footnotetext{
P5: We first take visual acuity. We may also take tension (IOP) based on the symptoms. The main test to lead us for further is visual acuity. [..]

I: How do you detect glaucoma here?

P5: Mostly it is clinical. If they complain about pain, we suspect glaucoma then we take tension (IOP). If the tension is above normal, we immediately refer them to Jimma. If they cannot afford to go to Jimma, we start medication for them here.
}

JUMC is the only specialized tertiary hospital for the entire region of Southwest Ethiopia, serving more than 4 million people. The hospital has a well-established eye unit with its own separate building and staff: three ophthalmologists, 13 ophthalmology residents, five optometrists, two ophthalmic nurses, two cataract surgeons and one refractionist. Compared to primary hospitals, the unit is comparatively well equipped with medical equipment such as a slit lamp, a biomicroscope, a laser capsulotomy and a VFT device.

Patients travel to JUMC from areas as far as $450 \mathrm{~km}$, across the border with South Sudan. Patients start queueing up in the waiting area of the unit around 5AM, although service only starts around 8AM. Given the large number of patients showing up each day, the unit limits the daily intake. Some patients end up waiting three or more days before they are seen. Despite the fact that it is a tertiary care hospital, it often acts as a primary care contact point for many of the patients.

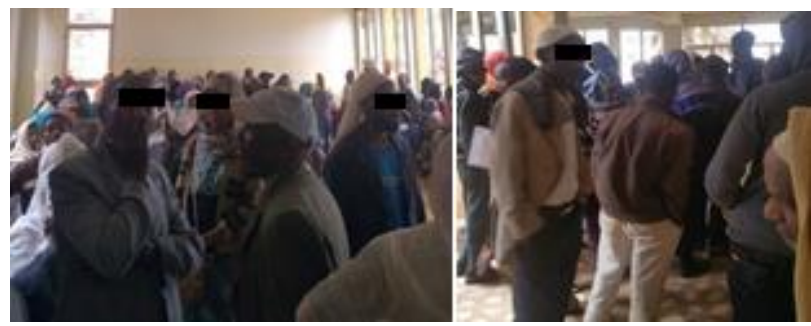


Figure 4: Left: Morning scene in the JUMC waiting room. Right: Other patients, outside the waiting room, unable to enter the overcrowded waiting room.

At the eye unit of JUMC, all new patients first go through basic screening tests. Patients with a suspected glaucoma take the visual field test using Frequency Doubling Technology (FDT) perimetry equipment. This a specialized but expensive medical device; however, even though it once comprised a gold standard, it has become obsolete in modern Western hospitals [32]. The Humphrey Field Analyzer (HFA) device, which is more accurate than the FDT, and currently considered the state of the art for visual field testing (and hence glaucoma diagnosis), is only available at a private clinic in the city of Jimma. Most patients visiting the eye unit of JUMC cannot afford to pay for private service, which is far more expensive than public health institutions. There is only one FDT device at this hospital, and there is no other public hospital in the Southwest where visual field-testing equipment is available.

\section{Outreach programs and NGOs}

This theme concerns the role of NGO's in the delivery of eye care. NGO's play a vital role in the effort to make eye care more accessible to rural communities by organizing and funding the outreach program of JUMC. Furthermore, they provide training to regular nurses on basic ophthalmic care, such as the treatment of TT.

Observations. The eye unit of JUMC, in collaboration with an NGO, organizes outreach missions to selected primary hospitals and health centres in the Southwest region to offer additional coverage of health services. As such initiatives depend, for example, on the current weather or the generosity of potential donors, they do not follow a consistent schedule. Yet, an average of seven missions can be realised each year. The main focus of these missions is cataract surgery, but other simple eye diseases are also treated on site. During outreach programs, test results are neither recorded nor printed out for further consultation.

On one such mission we observed at SaPH in August 2017, more than 115 patients were screened and, if necessary, received cataract surgery right away (see Figure 5). Every patient went through basic screening that involved simple tools such as torch light, a handheld ophthalmoscope, and a handheld tonometer. The screening tests take about ten minutes per patient with the aim to identify patients with cataracts, who require on-site surgery. For simpler health issues, such as infections, patients received medicine prescriptions, though for most conditions, including glaucoma, patients were advised to seek treatment at JUMC.
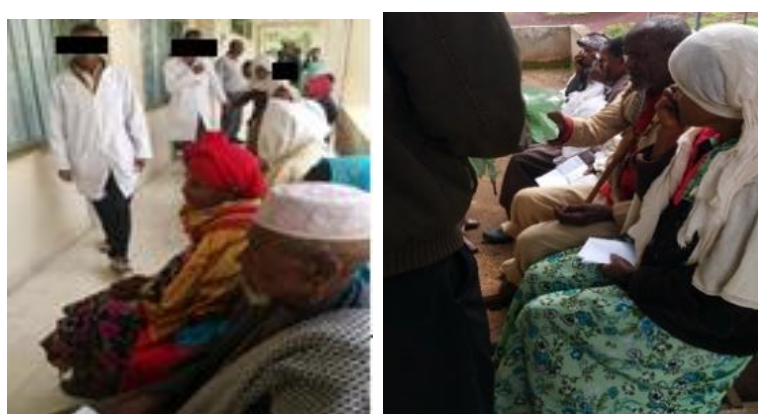

Figure 5. Outreach mission at Saja Primary

Hospital. Patients waiting for screening(Left).

Patients waiting to go through cataract surgery(Right).

Non-Governmental Organizations (NGOs) play a significant role in the organisation as well as the financing of outreach missions. For instance, the outreach program of JUMC is financed by the NGO "Light for the world". Another NGO, "Orbis International", is involved in the national "trachoma eradication" program [33] and has organized trainings for general nurses, focusing mainly on trachoma.

\footnotetext{
P6: "Every week on Wednesday and Thursday we move to more rural community households to conduct door-to-door eye exams. We mainly focus on trachoma."

I: Do you do this on a regular basis?

P6: "Yes, we are being supported by the NGO Orbis."
}

Both patients and health workers highly depend on the NGOs to fund and organize eye care. Health care workers are also expected to report to the funding NGO.

\footnotetext{
P6: We were mainly trained for trachoma. [...] We report quarterly to our donor and region office on the cases we have treated and referred.
}

The confidence in the NGO driven primary health care through outreach programs is high, to such an extent that the nurses we interviewed do not consider cost to be a critical factor for a portable glaucoma screening tool. They suggested NGOs will cover the expenses of such equipment.

\footnotetext{
I: Do you think a tool including the smartphone, VR headset and the remote would be affordable for your institution?

P6: "I think it is well affordable. Moreover, in addition to our hospital, NGOs can help us on this."

I: If there would have been a portable VFT tool, do you think visual field will also be taken during outreach?

P5: This depends on the objective of the campaign. Since the main objective of our outreach campaign is glaucoma screening, it would be done for sure. But the funding of this outreach is specifically for cataract. [...] The donors may not be happy if we don't focus on cataract.
}

\section{Glaucoma is too invisible to be a priority}

This theme concerns the relatively lower priority given to glaucoma compared to other eye diseases. This is mainly 
due to lack of more accurate prevalence data and asymptomatic nature of the disease. Other eye diseases such as cataracts and TT get higher priority both from the government and NGO's.

Observations. Glaucoma is currently not considered a major health problem. Other eye diseases are given a higher priority by the government and NGOs. In particular, TT has been the main focus of the IECW nurses in the primary hospitals. According to a national survey in 2006, the condition is the second cause of blindness in the country [3]. Moreover, different from glaucoma, symptoms are highly visible and easily identified with a torch light. Cataract is another eye condition slowly gaining attention in the primary hospitals, next to TT. Cataracts are also easily identifiable, compared to glaucoma. While not available through primary hospitals, outreach missions provide treatment to patients.

\section{P7: "The major focus is now on TT. There is a national program to eradicate TT. While we work on TT, we started to encounter cataract cases as well. Now, cataract is also becoming an issue of discussion. But we never hear anything regarding glaucoma. Maybe it could be a focus in the future after TT and cataracts?"}

However, according to ophthalmologists and ophthalmic nurses at JUMC, glaucoma is a very common condition among their patients. They believe it to be under-diagnosed and voiced frustration on the lack of interest from government and NGOs for this 'invisible disease'.

\section{P4: "Glaucoma is the least diagnosed disease in Ethiopia. There are a lot of cases that are missed. Some patients come here (at $J U M C$ ) complaining of other simple symptoms. But when we examine them, they already have advanced level of glaucoma. I have seen a patient with an IOP of 80. ."}

P2: "Glaucoma is very common case among patients in our clinic (JUMC). I don't think that the survey done some years ago, which is available online, reflects the real situation."

\section{Lack of prevalence data}

This theme concerns the lack of data on prevalence of eye diseases. The single most recent prevalence data on eye diseases in Ethiopia is from a national survey back in 2006. Ophthalmologists insist that eye disease such as glaucoma are actually more prevalent than what is reported in the survey.

Observations. In line with the lesser attention for 'invisible' eye diseases, data with respect to the prevalence rate of glaucoma in Southwest Ethiopia is similarly barely available. A national survey from 2006 puts glaucoma as the fifth major cause of blindness in Ethiopia [3]. However, the ophthalmologists we interviewed disagree with this number, and insist that based on the cases they encounter daily, glaucoma should be ranked higher. Unfortunately, there is no systematic way to collect data on the prevalence of various eye conditions.
However, at primary hospitals of SaPH and SCPH, the staff register all the cases on a form. Typically, they record the date, age, sex, condition and other details for each case. They use the record mainly for reporting purposes at the end of every month to NGOs. During outreach missions, Jimma university staff tallies each case identified by screening on a paper form, one for each day. Data is summarized by counting the tallied forms of all days at the end of the mission (see Figure 6). This process is entirely paper-based.

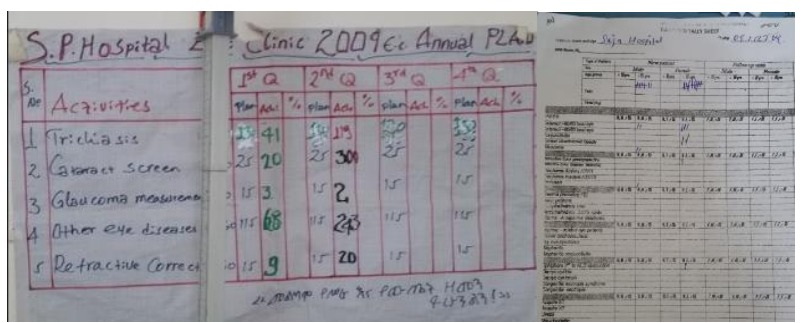

Figure 6. Paper forms for recording and reporting the number of different types of eye diseases.

\section{Problematic patient data storage and retrieval}

This theme concerns the current practices of patient record keeping and retrieval. Every patient information is kept in papers with a blue folder for each patient holding medical history including results of previous tests. Retrieving patient records for subsequent visits is a challenge to the extent where records of patients are missing.

Observations. In the hospitals we visited, there is no electronic medical record system; patient records are entirely paper-based. During outreach missions, patients show up for screening without registration. The screening results are not recorded. For diseases not treated at the site, patients are orally advised to go to JUMC. When patients are referred from a primary hospital like SaPH or SCPH to a tertiary hospital like JUMC, they are given a referral paper form, which is filled out by the referring institution with a summary of the medical details. The patient's folder is not exchanged between institutions.

\footnotetext{
I: When you refer to Jimma, what do you send with the patient? Do you include your test results?

P5: No, we just write a note that we suspect glaucoma. We don't send the results. We just send them with the standard referral form. Some patients come back from Jimma and tell us the medication they received from Jimma. Some don't come back, so we cannot follow them.
}

Upon registration at JUMC, every patient is assigned a new patient folder including details on name, age and address as well as their medical history and all test results (see Figure 7).

In the Ethiopian health care system, there is no social security provision that provides all patients with a unique identifiable number. Unfortunately, people from rural areas and older people often do not know their birth date and 
have an informal naming system. As a result, any system that identifies and retrieves patient records on a combination of name, birthdate or social security number is impossible. Hence, JMCU provides patients with a small paper card containing a unique identification number specific to the eye unit.

One of the major challenges with this paper-based patient record keeping system is retrieving patient folders during subsequent visits. Patients have to present the ID card to the reception so that their folders can be retrieved. Some patients lose the small card, making it difficult for the reception to locate their folders. But sometimes, even if the patient brings the ID card, folders can be missing. During our visit at JUMC, we witnessed a glaucoma patient whose medical record was not found, including results of three visual field tests taken previously.

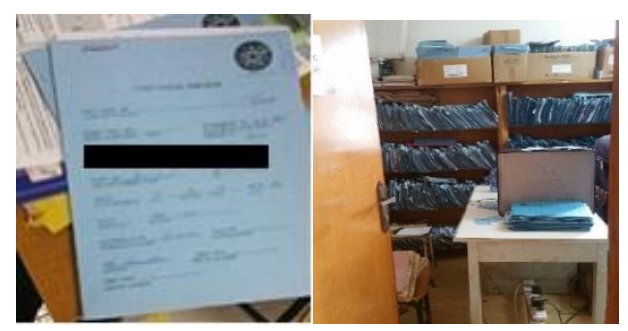

Figure 7 A blue folder holding patient records (Left).Patient folders on shelves behind the reception desk of the eye unit of JUMC (Right).

\section{Manual copying of test results}

This theme concerns the challenges ophthalmic nurses face in administering visual field tests using the FDT at JUMC. As the equipment had ran out of print paper, nurses are supposed to manually write down test results wasting more time and effort.

Observations. The FDT visual field test equipment at JUMC produces test results that can theoretically be printed. However, during our visit, the printer was out of order. The nurses told us it ran out of paper a long time ago. To store test results, they are copied manually from the device's screen on a paper form prepared for this purpose (see Figure 8). Ophthalmic professionals administering the visual field test spend much time and effort documenting results.

Power interruptions, which happen multiple times each day, are another challenge. Because the FDT equipment is not capable of saving intermediate test results, the entire test has to be repeated if power goes down before the test is completed and results have been copied onto paper. This is a constant worry of eye health workers and another reason why tests results are copied as quickly as possible on paper-a practice that is prone to errors.
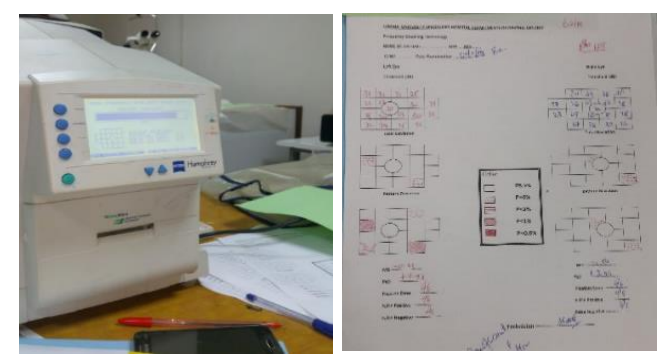

Figure 8. Various details of a test result are copied from the FDT screen to a paper form.

\section{Visual Field Testing is challenging for patients and nurses}

The last theme concerns patient related challenges ophthalmic nurses face in administering visual field test with FDT . As many of the patients performing the test are older adults from rural areas with little or no exposure to technology, more time and effort is spent in training the patients and repeating unsuccessful tests. As a consequence of this challenge, ophthalmic nurses consider it as a 'boring' task and tend to avoid it or at least delay it as much as possible.

Observations. At JUMC, a patient suspected of glaucoma based on basic screening tests (measuring IOP and inspection of the optic disc), may take the visual field test. During the test, stimuli appear at various locations of the visual field. The patient is expected to look at a fixation target at the centre of vision, for the entire duration of the test and to press a response button every time a stimulus is presented (see Figure 9) in the periphery. After a series of stimuli, the equipment compares the response pattern of the patient with a normative database to regions in the periphery with vision loss. The test is done separately for each eye [34].
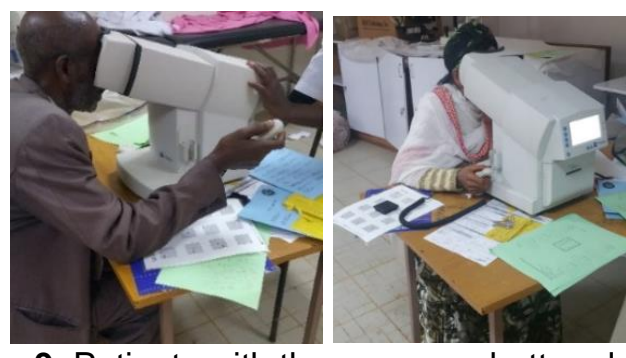

Figure 9. Patients with the response button during a visual field test with FDT.

Patient training is considered to be critical for the success of the test. A detailed explanation is given for those patients taking it for the first time. A leaflet from equipment manufacturer shows the fixation target and stimuli; nurses use it to prepare patients for the test (see Figure 10). In addition to the explanation by the ophthalmic professional, a demo test familiarizes patients with the test scene and response button, before the actual test begins. 


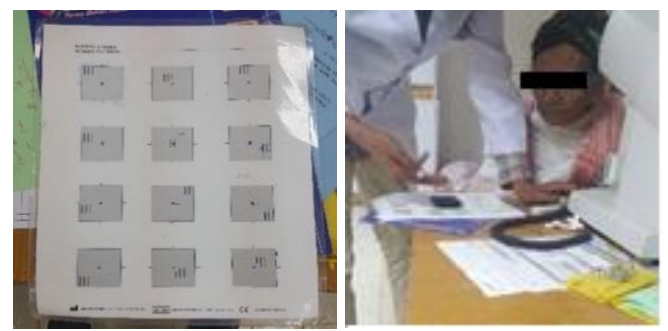

Figure 10. Left: FDT training manual with an illustration of stimuli. Right: An ophthalmic nurse is giving an explanation to a patient, using the manual.

The equipment relies on the age of the patient to determine the intensity of the stimulus presented, and to compare the results with a normative database. Most of the patients taking this test at JUMC are older adults from rural areas. Often they do not know their exact birth date, and cannot provide accurate age information which may affect the reliability of results. Furthermore, patients with an advanced level of glaucoma have damage in their central vision and are unable to fixate during the test. In the most advanced cases, patients are not be able to complete the test with their visual field results reported as, "cannot fixate".

$P_{4}$ : "For some patients we simply write "The patient cannot fixate".

So, they won 't have any FDT results."

Another challenge for older adults from rural areas is a lack of familiarity with technology. Understanding the visual field test procedure is difficult for them, especially during their first attempt. For most patients from rural areas, pressing the response button can by itself constitute a challenge.

Furthermore, patients highly respect physicians and are afraid of making mistakes. As one of the ophthalmic nurses mentioned, patients from rural areas consider medical professionals "next to God" and try to be as cooperative as possible. Because of this desire to please test examiners and the associated internalised shame, they might avoid reporting their difficulties during the visual field test. As a consequence, the test typically has to be repeated a number of times before a reliable outcome can be obtained. The entire testing session can take from 30 minutes up to more than an hour for some patients.

Given the challenges they encounter, particularly with older patients from rural areas, the ophthalmic nurses consider the test boring and tiresome work. Consequently, visual field tests are avoided in the morning, which are considered the busiest hours. Patients in need of VFT are scheduled for Friday, or in the afternoon. Both ophthalmic nurses we interviewed mentioned that they administer only three to five visual field tests per week. Moreover, to our surprise, we observed that the VFT room was closed during most of our visits, including Fridays.

\section{Discussion}

The themes that emerged from thematic analysis of the data contained pertinent information on the context of eye care in Southwest Ethiopia. They provided more insight into the organization of health care ,identified key stakeholders in the delivery of eye care, explained current practices in the screening and diagnosis of glaucoma and highlighted challenges faced by ophthalmic professionals and patients.

Upon further analysing the aforementioned themes, we were confronted with paradoxes - dynamics where apparently contradictory elements are interrelated to produce an unanticipated result. In the following paragraphs we detail these paradoxes, shed light on the causal mechanisms and suggest design opportunities for mobile health technologies addressing them.

\section{Paradox 1: Money is not an issue, yet there is no money for glaucoma}

We found both patients and health workers to be highly dependent on the NGOs to fund and organize eye care. Given the frequency and the reliability of NGO-organized and funded (outreach) programs, and the collaboration with national government, it can be argued that NGOs are an established part of primary and tertiary care. This is in line with other studies who described the important role of NGOs in the health sector in LICs [35].

The confidence in NGO driven primary health care is high, to such an extent that the nurses and doctors we interviewed did not consider cost to be a critical factor for a portable glaucoma screening tool. If the value of such a tool can be made 'tangible', they claim NGOs will cover the expenses. However, eye care professionals pointed out that NGOs (in agreement with national government) define what gets funded, and that the current focus is on TT and Cataract, not on glaucoma. Eye care workers are also expected to report the number of cases of TT and Cataract treated to the NGOs and government. Health care programs are driven by impact, and governments as well need figures on prevalence and ailments. Detrimental impact of diseases as well as the positive impact through campaigns need to be made tangible [36]. Glaucoma is currently not 'visible'. Despite the conviction of the ophthalmologists that it is highly prevalent and detrimental.

Hence, design opportunities for mobile eye health care solutions could consider features that enhance 'visibility of impact' such as storing the times it was used, documenting the number of health centers where it was used, recording the number of patients helped, etc.

We also witnessed issues surrounding the base data tests are conducted on. The FDT test results are compared to a normative dataset from Western patients, despite the fact that the prevalence of Glaucoma is higher on the African continent, and African patients may differ in progress of the condition [37]. Despite the lack of representation, these normative datasets are specific to a given piece of 
equipment and cannot be reused on a different equipment with different parameters [38].

Hence, design opportunities for mobile eye health care solutions lie in screening tests that require reference database of normal subjects, such as the VFT screening. Researchers and designers could investigate how to collect and store results with the aim to build localised reference datasets.

\section{Paradox 2: Shortage of eye health care, yet the work is repeated over and over}

The ophthalmologists-to-population ratio in Ethiopia is one per million people [15], in sharp contrast with the high prevalence of eye diseases. Based on our interviews and observations, no ophthalmologist doctor can diagnose and perform surgery in any other hospital in the Southwest of Ethiopia, except at JUMC. As a consequence, patients need to travel long distances, in difficult circumstances, and wait sometimes up to three (3!) days for an initial consultation.

Despite this shortage in eye health care professionals, we observed many inefficient work practices. A first loss in efficiency is rooted in the combination of a paper-based record system and the absence of a national social security or citizen identification program. As a consequence, each health institution maintains its own paper-based patient record, and patients (also illiterate patients from rural areas) are responsible for retaining their unique identifier number, printed on a small paper slip. This results in a broken information flow, i.e. a lack exchange of patient data among hospitals. Results from earlier tests at other hospitals or during outreach missions are not (re-)used. Moreover, patients lose their ID card or paper folders are misplaced and no longer found.

Patient data exchange is generally challenging-even in high-income countries [39]. Given the absence of electronic health records and national patient identification, mobile health solutions cannot take advantage of linking data to existing electronic health records.

The design of the visual field-testing equipment itself is also a cause of superfluous work. Power outages and unsteady access to internet, in combination with a device that cannot save intermediate results, lead to visual field tests having to be performed repeatedly. Moreover, an unpredictable supply of print paper complicates printer usage. Hence, test results are manually copied from the device's screen onto a paper form. As a result, ophthalmic nurses find VFT a lengthy, tedious job. While it is essential for diagnosis, we witnessed it was avoided as much as possible. The fact that eye health professionals would rather rely on other inferior tests for the diagnosis of glaucoma, is a practical example of how technology can end up being under-utilized if not designed to fit in to the actual context [40].

The finding of unreliable IT infrastructure may contrast with the high rate of penetration of mobile phones and rapid expansion in coverage of the underlying network infrastructure in LICs [41]. We did find that, most of the time, ICT is up and running in rural Ethiopia. However, we also found short moments of power outages are frequent, which affects the stationary equipment in hospitals more than personal mobile devices.

Hence, design opportunities for mobile eye health care solutions could lie in the combination of taking advantage of the presence of ICT infrastructure, without being stymied by the frequent glitches. They should be able to continue to work in periods of temporary downtime of communication networks, i.e. through implementations of good syncing protocols as well as online and offline modes. Furthermore, they should be able to work in periods without electricity, i.e. by battery management and powerand back up strategies and avoid relying on third party logistics, such as printers, copiers, etc.

\section{Paradox 3: The willing but uncooperative patient tested by the willing but unexperienced nurse}

As a final paradox, we found that the patients and nurses themselves also pose a particular challenge. First, for many health solutions, similar to VFT, participation from the patient is indispensable. Therefore, the patient needs a clear understanding of the test and what they need to do, to obtain a reliable result. As seen from the experience with the FDT at JUMC, old patients from rural areas have difficulties in understanding the test, mainly due to lack of familiarity with technology. If a portable visual field screening tool is to be used in situations like the outreach mission, this issue is even more pronounced.

Hence, design opportunities for mobile eye health care solutions are in the field of on boarding and training so that patients can gain experience with tests before taking them, e.g. through a tutorial or entry level. They should also be accompanied by instructive materials or demos, that make the explanation of the test easier for patients, e.g. manuals, demonstrations, etc.

Due to high respect for medical doctors and social desirability, patients do not dare to freely share problems or discomfort. This lack in straightforward communication can result in skewed or unusable test results.

Hence, design opportunities for mobile eye health care solutions could allow test examiners to closely monitor the patient's performance, instead of having to rely on their verbalisations. Examiners should also be given access to intermediate test results to check and easily abort and restart tests if necessary.

\section{Conclusion}

We presented a qualitative analysis of the organization of eye care in Southwest Ethiopia, mainly focusing on screening for glaucoma. Through an inductive thematic 
analysis on the basis of semi-structured interviews and observations made at three different hospitals, we have been able to highlight the challenges faced by ophthalmic professionals and patients, and identified paradoxes in the practice of eye care that present design opportunities. Our findings bear the potential to inspire future designs of mobile health solutions targeting rural communities in Ethiopia and other LICs.

\section{Acknowledgements.}

The authors thank the department of Ophthalmology of Jimma University for their support during data collection. We also thank all the participants for dedicating their valuable time to the interviews and observations.

\section{References}

[1] WHO Action Plan for the Prevention of Avoidable Blindness and Visual Impairment 2009-2013. (n.d.). IAPB, from https://www.iapb.org/resources/who-action-planfor-the-prevention-of-avoidable-blindness-and-visualimpairment-2009-2013/, accessed 19-3-2018

[2] Thylefors, B. (1998). A GLOBAL INITIATIVE FOR THE ELIMINATION OF AVOIDABLE BLINDNESS, Community Eye Health, Vol. 11, No. 25, 1-3

[3] Berhane, Y.; Worku, A.; Bejiga, A.; Adamu, L.; Alemayehu, W.; Bedri, A.; Haile, Z.; Ayalew, A.; Adamu, Y.; Gebre, T.; Kebede, T. D.; West, E.; West, S. (2007). Prevalence and causes of blindness and Low Vision in Ethiopia, Ethiopian Journal of Health Development, Vol. 21, No. 3, 204-210. doi:10.4314/ejhd.v21i3.10050

[4] Zerihun, N.; Mabey, D. (1997). Blindness and low vision in Jimma Zone, Ethopia: results of a population-based survey, Ophthalmic Epidemiology, Vol. 4, No. 1, 19-26. doi:10.3109/09286589709058057

[5] Parihar, J. K. S. (2016). Glaucoma: The 'Black hole' of irreversible blindness, Medical Journal, Armed Forces India, Vol. 72, No. 1, 3-4. doi:10.1016/j.mjafi.2015.12.001

[6] Accuracy of referral to a glaucoma clinic - Bell - 1997 Ophthalmic and Physiological Optics - Wiley Online Library. (n.d.), from https://onlinelibrary-wileycom.kuleuven.ezproxy.kuleuven.be/doi/abs/10.1046/j.147 5-1313.1997.9600066x.x, accessed 28-9-2018

[7] Abate, A.; Kifle, M.; Okuboyejo, S.; Mbarika, V. (2017). A mobile-based telepathology system for a low resource setting in Ethiopia, Applied Computing and Informatics. doi:10.1016/j.aci.2017.08.002

[8] Ginsburg, A. S.; Agyemang, C. T.; Ambler, G.; Delarosa, J.; Brunette, W.; Levari, S.; Larson, C.; Sundt, M.; Newton, S.; Borriello, G.; Anderson, R. (2016). mPneumonia, an Innovation for Diagnosing and Treating Childhood Pneumonia in Low-Resource Settings: A Feasibility, Usability and Acceptability Study in Ghana, PLOS ONE, Vol. 11, No. 10, e0165201. doi:10.1371/journal.pone.0165201

[9] Gallay, C.; Girardet, A.; Viviano, M.; Catarino, R.; Benski, A.-C.; Tran, P. L.; Ecabert, C.; Thiran, J.-P.; Vassilakos, P.; Petignat, P. (2017). Cervical cancer screening in low-resource settings: a smartphone image application as an alternative to colposcopy, International
Journal of Women's Health, Vol. 9, 455-461. doi:10.2147/IJWH.S136351

[10] Ehsan, U.; Sakib, N.; Haque, M. M.; Soron, T.; Saxena, D.; Ahamed, S.; Schwichtenberg, A.; Rabbani, G.; Akter, S.; Alam, F.; Begum, A.; Ahmed, S. (2018). Confronting Autism in Urban Bangladesh: Unpacking Infrastructural and Cultural Challenges, EAI Endorsed Transactions on Pervasive Health and Technology, Vol. 4, No. 14

[11] Aranda-Jan, C. B.; Mohutsiwa-Dibe, N.; Loukanova, S. (2014). Systematic review on what works, what does not work and why of implementation of mobile health (mHealth) projects in Africa, BMC Public Health, Vol. 14, 188. doi:10.1186/1471-2458-14-188

[12] Johnson, C. M.; Johnson, T. R.; Zhang, J. (2005). A usercentered framework for redesigning health care interfaces, Journal of Biomedical Informatics, Vol. 38, No. 1, 75-87. doi:10.1016/j.jbi.2004.11.005

[13] What Is Glaucoma? (2017, March 1). American Academy of Ophthalmology, from https://www.aao.org/eyehealth/diseases/what-is-glaucoma, accessed 22-1-2018

[14] Primary Open-Angle Glaucoma. (n.d.). All About Vision, from https://www.allaboutvision.com/conditions/primaryopen-angle-glaucoma.htm, accessed 4-10-2018

[15] Melese, M.; Alemayehu, W.; Friedlander, E.; Courtright, P. (2004). Indirect costs associated with accessing eye care services as a barrier to service use in Ethiopia, Tropical Medicine \& International Health, Vol. 9, No. 3, 426-431. doi:10.1111/j.1365-3156.2004.01205.x

[16] Federal Democratic Republic of Ethiopia Ministry of Health: Health Sector Development Program IV 2010/112014/15. (n.d.). Healthy Newborn Network, from https://www.healthynewbornnetwork.org/resource/federal -democratic-republic-ethiopia-ministry-health-healthsector-development-program-iv-201011-201415/, accessed 5-12-2017

[17] WHO | Ethiopia. (n.d.). WHO, from https://www.who.int/countries/eth/coop_strategy/en/inde x1.html, accessed 12-1-2019

[18] Latif, S.; Rana, R.; Qadir, J.; Ali, A.; Imran, M. A.; Younis, M. S. (2017). Mobile Health in the Developing World: Review of Literature and Lessons From a Case Study, IEEE Access, Vol. 5, 11540-11556. doi:10.1109/ACCESS.2017.2710800

[19] Giorgis, A. T. (2012). Raising public awareness of glaucoma in Ethiopia, Community Eye Health, Vol. 25, Nos. 79-80, 46

[20] Haymes, S. A.; LeBlanc, R. P.; Nicolela, M. T.; Chiasson, L. A.; Chauhan, B. C. (2007). Risk of Falls and Motor Vehicle Collisions in Glaucoma, Investigative Ophthalmology \& Visual Science, Vol. 48, No. 3, 11491155. doi:10.1167/iovs.06-0886

[21] Ayele, F. A.; Zeraye, B.; Assefa, Y.; Legesse, K.; Azale, T.; Burton, M. J. (2017). The impact of glaucoma on quality of life in Ethiopia: a case-control study, $B M C$ Ophthalmology, Vol. 17. doi:10.1186/s12886-017-0643-8

[22] Infeld, D. A.; O'Shea, J. G. (1998). Glaucoma: diagnosis and management., Postgraduate Medical Journal, Vol. 74, No. $878,709-715$

[23] Kumar, S.; Giubilato, A.; Morgan, W.; Jitskaia, L.; Barry, C.; Bulsara, M.; Constable, I. J.; Yogesan, K. (2007). Glaucoma screening: analysis of conventional and telemedicine-friendly devices, Clinical \& Experimental Ophthalmology, Vol. 35, No. 3, 237-243. doi:10.1111/j.1442-9071.2007.01457.x

[24] Tak, A.; Ustun, T. S. (2016). Wireless power grid: Leapfrogging in power infrastructure of developing 
countries, 2016 IEEE Region 10 Conference (TENCON)Presented at the 2016 IEEE Region 10 Conference (TENCON), , 1274-1277. doi:10.1109/TENCON.2016.7848217

[25] Moradian, S.; Safi, S. (2015). Application of Mobile Phones in Ophthalmology, Journal of Ophthalmic \& Vision Research, Vol. 10, No. 2, 200-205. doi:10.4103/2008-322X.163775

[26] Lord, R. K.; Shah, V. A.; Filippo, A. N. S.; Krishna, R. (2010). Novel Uses of Smartphones in Ophthalmology, Ophthalmology, Vol. 117, No. 6, 1274-1274.e3. doi:10.1016/j.ophtha.2010.01.001

[27] Bastawrous, A.; Leak, C.; Howard, F.; Kumar, V. (2012). Validation of Near Eye Tool for Refractive Assessment (NETRA) - Pilot Study, Journal of Mobile Technology in Medicine, Vol. 1, No. 3, 6-16

[28] Burton, M.; Habtamu, E.; Ho, D.; Gower, E. W. (2015). Interventions for trachoma trichiasis, The Cochrane Database of Systematic Reviews, No. 11. doi:10.1002/14651858.CD004008.pub3

[29] Noy, C. (2008). Sampling Knowledge: The Hermeneutics of Snowball Sampling in Qualitative Research, International Journal of Social Research Methodology, Vol. 11, No. 4, 327-344. doi:10.1080/13645570701401305

[30] Braun, V.; Clarke, V. (2006). Using thematic analysis in psychology, Qualitative Research in Psychology, Vol. 3, No. 2, 77-101

[31] Beyer, H.; Holtzblatt, K. (1998). Contextual Design: Defining Customer-Centered Systems, Morgan Kaufmann Publishers Inc., San Francisco, CA, USA

[32] Tafreshi, A.; Racette, L.; Weinreb, R. N.; Sample, P. A.; Zangwill, L. M.; Medeiros, F. A.; Bowd, C. (2010). Pattern Electroretinogram and Psychophysical Tests of Visual Function for Discriminating Between Healthy and Glaucoma Eyes, American Journal of Ophthalmology, Vol. 149, No. 3, 488-495. doi:10.1016/j.ajo.2009.09.027

[33] Habtamu, E.; Rajak, S. N.; Gebre, T.; Zerihun, M.; Genet, A.; Emerson, P. M.; Burton, M. J. (2011). Clearing the Backlog: Trichiasis Surgeon Retention and Productivity in Northern Ethiopia, PLOS Neglected Tropical Diseases, Vol. 5, No. 4, e1014. doi:10.1371/journal.pntd.0001014

[34] Johnson, C. A. (2008). FDT perimetry for the detection of glaucomatous visual field loss, J Glaucoma Today, 26-28

[35] Wamai, R. G. (2008). Reforming Health Systems: The Role of NGOs in Decentralization: Lessons from Kenya and Ethiopia, International society for third-sector research (ISTR)

[36] Mueller-Hirth, N. (2012). If You Don't Count, You Don't Count: Monitoring and Evaluation in South African NGOs, Development and Change, Vol. 43, No. 3, 649670. doi:10.1111/j.1467-7660.2012.01776.x

[37] Lim, E.; Miyamura, J.; Chen, J. J. (2015). Racial/EthnicSpecific Reference Intervals for Common Laboratory Tests: A Comparison among Asians, Blacks, Hispanics, and White, Hawai' 'i Journal of Medicine \& Public Health, Vol. 74, No. 9, 302-310

[38] Lyne Racette, Monika Fischer, Hans Bebie,; Gábor Holló, Chris A. Johnson, Chota Matsumoto. (2016). Visual Field Digest (6th Edition.)

[39] Vest, J. R.; Gamm, L. D. (2010). Health information exchange: persistent challenges and new strategies, Journal of the American Medical Informatics Association, Vol. 17, No. 3, 288-294. doi:10.1136/jamia.2010.003673

[40] Brewer, E.; Demmer, M.; Du, B.; Ho, M.; Kam, M.; Nedevschi, S.; Pal, J.; Patra, R.; Surana, S.; Fall, K. (2005).
The case for technology in developing regions, Computer, Vol. 38, No. 6, 25-38. doi:10.1109/MC.2005.204

[41] Aker, J. C.; Mbiti, I. M. (2010). Mobile Phones and Economic Development in Africa, Journal of Economic Perspectives, Vol. 24, No. 3, 207-232. doi:10.1257/jep.24.3.207 